\title{
(Near-Infrared) Fluorescence-Guided Surgery Under Ambient Light Conditions: A Next Step to Embedment of the Technology in Clinical Routine
}

\author{
Nynke S. van den Berg, MSc ${ }^{1,2}$, Mitsuharu Miwa, BE ${ }^{3}$, Gijs H. KleinJan, MD ${ }^{1,4}$, Takayuki Sato, BE ${ }^{3}$, \\ Yoshiki Maeda, BE ${ }^{5}$, Alexander C. J. van Akkooi, MD, $\mathbf{P h D}^{6}$, Simon Horenblas, MD, $\mathbf{P h D}^{2}$, Baris Karakullukcu, \\ $\mathrm{MD}, \mathrm{PhD}^{7}$, and Fijs W. B. van Leeuwen, $\mathrm{PhD}^{1,2,7}$
}

${ }^{1}$ Interventional Molecular Imaging Laboratory, Department of Radiology, Leiden University Medical Center, Leiden, The Netherlands; ${ }^{2}$ Department of Urology, The Netherlands Cancer Institute - Antoni van Leeuwenhoek Hospital, Amsterdam, The Netherlands; ${ }^{3}$ Business Incubator, Development Center, Hamamatsu Photonics K.K., Hamamatsu, Japan; ${ }^{4}$ Department of Nuclear Medicine, The Netherlands Cancer Institute - Antoni van Leeuwenhoek Hospital, Amsterdam, The Netherlands; ${ }^{5}$ Planning and Project Group, Business Planning and Development, Hamamatsu Photonics K.K., Hamamatsu, Japan; ${ }^{6}$ Department of Surgical Oncology, The Netherlands Cancer Institute - Antoni van Leeuwenhoek Hospital, Amsterdam, The Netherlands; ${ }^{7}$ Department of Head and Neck Surgery and Oncology, The Netherlands Cancer Institute Antoni van Leeuwenhoek Hospital, Amsterdam, The Netherlands

\begin{abstract}
Background and Purpose. In open surgery procedures, after temporarily dimming the lights in the operation theatre, the Photo Dynamic Eye (PDE) fluorescence camera has, amongst others, been used for fluorescence-guided sentinel node ( $\mathrm{SN}$ ) biopsy procedures. To improve the clinical utility and logistics of fluorescence-guided surgery, we developed and evaluated a prototype modified PDE (mPDE) fluorescence camera system.

Methods. The m-PDE works under ambient light conditions and includes a white light mode and a pseudo-greencolored fluorescence mode (including a gray-scaled anatomical background). Twenty-seven patients scheduled for SN biopsy for (head and neck) melanoma $(n=16)$, oral cavity $(n=6)$, or penile $(n=5)$ cancer were included. The number and location of SNs were determined following an indocyanine green- ${ }^{99 \mathrm{~m}} \mathrm{Tc}$-nanocolloid
\end{abstract}

Electronic supplementary material The online version of this article (doi:10.1245/s10434-016-5186-3) contains supplementary material, which is available to authorized users.

(C) The Author(s) 2016. This article is published with open access at Springerlink.com

First Received: 29 November 2015;

Published Online: 28 March 2016

F. W. B. van Leeuwen, $\mathrm{PhD}$

e-mail: F.W.B.van_Leeuwen@lumc.nl injection and preoperative imaging. Intraoperatively, fluorescence guidance was used to visualize the SNs. The mPDE and conventional PDE were compared head-to-head in a phantom study, and in seven patients. In the remaining 20 patients, only the m-PDE was evaluated.

Results. Phantom study: The m-PDE was superior over the conventional PDE, with a detection sensitivity of $1.20 \times 10^{-11} \mathrm{M}$ (vs. $3.08 \times 10^{-9} \mathrm{M}$ ) ICG in human serum albumin. In the head-to-head clinical comparison $(n=7)$, the m-PDE was also superior: (i) $\mathrm{SN}$ visualization: 100 versus $81.4 \%$; (ii) transcutaneous SN visualization: 40.7 versus $22.2 \%$; and (iii) lymphatic duct visualization: 7.4 versus $0 \%$. Findings were further underlined in the 20 additionally included patients.

Conclusion. The m-PDE enhanced fluorescence imaging properties compared with its predecessor, and provides a next step towards routine integration of real-time fluorescence guidance in open surgery.

Different groups have reported that for effective intraoperative (near-infrared) fluorescence imaging the lights in the operating room have to be dimmed, or switched off, in order to visualize the fluorescence signal. ${ }^{1,2}$ This results in temporary stalling of the surgical procedure, even when the fluorescence camera itself is equipped with a white light source. ${ }^{2}$ Therefore, in general, the fluorescence guidance technology is primarily used to provide static confirmatory information regarding the location of lesions. ${ }^{3}$ Ideally, 
during a surgical procedure the technique would be used to allow the surgeon to excise the lesion of interest under realtime fluorescence guidance.

Previously, in laparoscopic studies using the hybrid tracer indocyanine green (ICG) ${ }^{99 \mathrm{~m}} \mathrm{Tc}$-nanocolloid, we showed that the value of real-time fluorescence guidance significantly increased when the fluorescent signal was displayed within the anatomical context of the patient. ${ }^{4}$ For open surgery procedures, using the Photo Dynamic Eye fluorescence camera (PDE; Hamamatsu Photonics K.K., Hamamatsu, Japan), we saw that in some cases the background signal helped provide anatomical context. ${ }^{5-7} \mathrm{We}$ reasoned that exploiting this feature further could aid the routine embedment of the technology. Allowing fluorescence guidance under ambient light conditions would, at the same time, help simplify clinical logistics. To achieve our goals, we set out to develop a prototype modified PDE (m-PDE) fluorescence camera, and evaluated it in both a phantom and patient study.

\section{MATERIALS AND METHODS}

\section{Fluorescence Camera Systems}

We evaluated the newly developed prototype m-PDE fluorescence camera and compared it to the commercially available conventional PDE (c-PDE) fluorescence camera (Hamamatsu Photonics K.K., Hamamatsu, Japan).

The main differences between the c-PDE and the m-PDE are shown in Table 1. Briefly, the light-emitting diode (LED)based near-infrared excitation light of the c-PDE works in a continuous wave mode, while the illumination source of the $\mathrm{m}$-PDE is pulsed in synchronization with the frame rate of the charge-coupled device (CCD). Here, pulsation means the CCD detector obtains both a fluorescence image containing ambient light background signal and an image of the ambient light background only. Real-time subtraction of the two images then allows the m-PDE to obtain a 'pure' fluorescence image (in gray-scale or pseudo-green-color) under ambient light conditions. Second, the m-PDE also allows real-time mixing of the 'pure' pseudo-green-colored fluorescence image with the gray-scale anatomical context image. As a third improvement, the m-PDE can also show a white light image in a non-fluorescence imaging setting.

\section{Phantom Study}

A $5.0 \mathrm{mg} / \mathrm{mL}\left(6.45 \times 10^{-3} \mathrm{M}\right)$ ICG (ICG-Pulsion, 25 mg vial; Pulsion Medical Systems, Munich, Germany)human serum albumin (HSA; Albuman 200 g/L; Sanquin, Amsterdam, The Netherlands) solution was prepared and diluted $1: 1$ with HSA in 30 steps down to $9.31 \mathrm{ng} / \mathrm{mL}$ $\left(1.20 \times 10^{-11} \mathrm{M}\right)$. From each dilution $100 \mu \mathrm{L}$ was pipetted in a black 96-well plate (Cellstar; Greiner Bio-One $\mathrm{GmbH}$, Frickenhausen, Germany). The complete dilution range was then evaluated to determine the detection sensitivity of the m-PDE and c-PDE fluorescence camera systems. Hereby, the head of the fluorescence cameras was fixed, perpendicular, at a $14 \mathrm{~cm}$ distance from the wellplate surface. This allowed capture of the whole dilution range in the field of view.

Imaging of the plate was performed under different settings-white light (m-PDE only) and fluorescence (both systems) and under various light conditions: (i) all lights in the operating room turned on (halogen satellite lamps directly lighting the sterile field [angle of approximately $45^{\circ}$ with regard to the plate surface], the plenum and surrounding lights [both tubular lights]); (ii) satellite lamps directly lighting the sterile field turned off, but the plenum and surrounding lights on (referred to as 'ambient light' conditions); and (iii) all lights in the operating room dimmed. For the m-PDE fluorescence camera system evaluation, in all experiments the pseudocolored green setting was used.

As a reference for the fluorescence intensity measured with the c-PDE and m-PDE fluorescence camera systems, the ICG-based dilution range was also measured on preclinical, cooled, black box, camera systems (IVIS Spectrum, Xenogen Corporation, San Francisco, CA, USA; and the Pearl Impulse, LI-COR Biotechnology GmbH, Hombur, Germany). The fluorescence image obtained with the IVIS Spectrum was presented in a pseudo-colored glow scale, whereas for the Pearl Impulse, the fluorescence signal was presented in a pseudo-colored green scale. For both systems, the fluorescence images were overlaid onto a black and white background image.

For quantification of the fluorescence signal measured with the IVIS Spectrum, in the acquired fluorescence image, regions of interest were drawn surrounding the wells after which Living Image 3D analysis software (version 1.0; Xenogen Corporation) was used to quantify the signal intensity per well.

\section{Light Spectra Measurements}

Light spectra of the different lamps present in the operating room were determined using a Jobin Yvon VS140 linear array fiber spectrometer (Horiba, Kyoto, Japan) in the 300-1200 nm range, with an integration time of $0.1 \mathrm{~ms}$. The fiber was held at a 2-meter distance from the lamp from which the light spectra were measured.

\section{Absorption and Emission Spectra Measurements of ICG-HSA}

The absorption and emission spectrum of ICG-HSA (concentration: $1.5 \times 10-9 \mathrm{M}$ ) was measured using an 
TABLE 1 Characteristics of the conventional and modified PDE fluorescence cameras

\begin{tabular}{lll}
\hline & c-PDE & m-PDE \\
\hline Excitation light source & LED (continuous) & LED (pulsed) \\
Imaging device & CCD & CCD \\
Excitation/emission wavelength & $760 />820 \mathrm{~nm}$ & $760 />820 \mathrm{~nm}$ \\
Handheld & Yes & Yes \\
Pulsed fluorescence imaging & No & Yes \\
White-light imaging & No & Yes \\
Focus adjustment & No & Yes \\
Effective under ambient light conditions & No & Yes \\
Pseudocoloring & No & Yes (green) \\
Fluorescence image presented in & Black and white & 1. Black and white \\
& & 2. Pseudocolored green on a gray- \\
\hline
\end{tabular}

$P D E$ Photo Dynamic Eye, $L E D$ light-emitting diode, $C C D$ charge-coupled device

Ultrospec $3000 \mathrm{UV} / \mathrm{Vis}$ spectrophotometer (Pharmacia Biotech/GE Healthcare Europe GmbH, Eindhoven, The Netherlands) and an LS55 fluorescence spectrometer (PerkinElmer, Groningen, The Netherlands). Solutions were prepared in a $3 \mathrm{~mL}$ quartz cuvet (Hellma $\mathrm{GmbH} \&$ Co. KG, Müllheim, Germany).

\section{Patient Study}

Patients Patients with squamous cell carcinoma of the oral cavity $(n=6)$ or penis $(n=5)$, head and neck melanoma $(n=11)$, or melanoma on the trunk or on an extremity $(n=5)$ scheduled for sentinel node (SN) biopsy with subsequent treatment of the primary tumor/re-excision of the melanoma scar were prospectively enrolled after obtaining written informed consent. All included patients were clinically node-negative as defined by palpation and ultrasound-guided fine needle aspiration cytology. Patient characteristics are shown in Table 2. The study protocol was conducted in accordance with the Helsinki Declaration and approved by the Medical Ethical Committee of the Dutch Cancer Institute - Antoni van Leeuwenhoek Hospital.

\section{Hybrid Tracer Preparation, Administration,} Preoperative Sentinel Node Mapping and (Histo-) Pathology

Preparation and administration of the hybrid tracer ICG- ${ }^{99 \mathrm{~m}}$ Tc-nanocolloid, preoperative imaging, and (histo-) pathological specimen analysis for oral cavity cancer, ${ }^{6}$ penile cancer, ${ }^{8}$ and (head and neck) melanoma ${ }^{7}$ have been previously described.

Surgical Procedure In patients with head and neck malignancies, primary tumor removal or re-excision of the melanoma scar was completed prior to performing $\mathrm{SN}$ biopsy. In penile cancer patients and patients with a melanoma on the trunk or on an extremity, SN biopsy was performed prior to treatment of the primary tumor site or the melanoma scar. A schematic overview of the intraoperative SN excision procedure is given in Fig. 1.

\section{RESULTS}

\section{Phantom Study}

Reference Fluorescence Data Figure 2a illustrates the relation between the ICG-HSA concentration and the fluorescence intensity measured with the IVIS Spectrum. Under black-box conditions, the lowest concentration evaluated $\left(1.20 \times 10^{-11} \mathrm{M}\right.$ ICG-HSA) could be easily detected using this system (Fig. 2b). The Pearl Impulse showed a similar detection range (data not shown).

Spectral analysis of the light emissions encountered for the different light settings evaluated in the operating room (Fig. 2c) revealed that the light spectrum of the (halogen) satellite lamps gives a broad emission spectrum that shows significant overlap with the spectral area where the ICG emission is collected. The severity with which the satellite lamps influenced ICG detection depended on the angle under which the satellite lamp was placed relative to the phantom. Hereby, the sensitivity for ICG was highest when the satellite lamp was angled so that the reflected satellite lamplight did not align with the position of the fluorescence camera. The normal surrounding lamps (tubular lights) displayed an assembly of light peaks, with the most pronounced emission maxima at 545 and $612 \mathrm{~nm}$, which showed a limited degree of spectral overlap with the emission peak of ICG (Fig. 2c, d). 
TABLE 2 Pre- and intraoperative sentinel node identification findings and pathology results

\begin{tabular}{|c|c|c|c|}
\hline & \multicolumn{2}{|c|}{ Direct camera comparison } & \multirow[t]{2}{*}{ Evaluation of $\mathrm{m}-\mathrm{PDE}$} \\
\hline & c-PDE & m-PDE & \\
\hline \multicolumn{4}{|l|}{ Patient characteristics } \\
\hline Patients & 7 & & 20 \\
\hline Average age, years (range) & $64.6(58-74)$ & & $54.4(34-81)$ \\
\hline Male/female ratio & $5 / 2$ & & $14 / 6$ \\
\hline \multicolumn{4}{|l|}{ Tumor type + tumor stage } \\
\hline SCC, oral cavity & 4 & & 2 \\
\hline $\mathrm{T} 1$ & 4 & & 2 \\
\hline Melanoma (head and neck, trunk, or extremity) & $3(1,1,1)$ & & $13(10,1,2)$ \\
\hline Average Breslow thickness, mm (range) & $1.6(1.2-2.1)$ & & $2.1(0.6-4.0)$ \\
\hline Ulceration, yes/no & $0 / 3$ & & $3 / 10$ \\
\hline SCC, penis & - & & 5 \\
\hline $\mathrm{T} 1$ & - & & 2 \\
\hline $\mathrm{T} 2$ & - & & 3 \\
\hline \multicolumn{4}{|l|}{ Preoperative SN mapping } \\
\hline Average injected dose, MBq (range) & $69.6(62.1-77.1)$ & & $80.6(67.3-156)$ \\
\hline $\begin{array}{l}\text { Preoperative number of SNs identified using } \\
\text { SPECT/CT (average, range) }\end{array}$ & $21(3,2-5)$ & & $51(2.6,1-6)$ \\
\hline No. of basins (\% total), no. of SNs (\% total) & $16(100), 21(100)$ & & $40(100), 51(100)$ \\
\hline Head & $1(6.3), 1(4.8)$ & & $5(12.5), 6(11.8)$ \\
\hline Auricular & - & & - \\
\hline Parotid gland & - & & $2(5.0), 3(5.9)$ \\
\hline Neck (level I-V) & $11(68.8), 15(71.4)$ & & 19 (47.5), $23(45.1)$ \\
\hline Axilla & $2(12.5), 2(9.5)$ & & $2(5.0), 2(3.9)$ \\
\hline Supraclavicular & - & & $1(2.5), 1(2.0)$ \\
\hline Scapular & $1(6.3), 1(4.8)$ & & - \\
\hline Groin & $1(6.3), 2(9.5)$ & & $11(27.5), 16(31.4)$ \\
\hline Average time injection - operation, hrs (range) & $5.5(4.3-6.5)$ & & $6.4(3.5-19.5)$ \\
\hline \multicolumn{4}{|c|}{ Intraoperative SN Identification } \\
\hline $\begin{array}{l}\text { No. of intraoperatively excised SNs (average, } \\
\text { range) }\end{array}$ & $27(3.9,2-7)$ & & $73(3.7,1-7)$ \\
\hline Radioactive & 27 & & 73 \\
\hline Fluorescent & 27 & & 73 \\
\hline Blue & $1^{\mathrm{a}}$ & & $12^{\mathrm{b}}$ \\
\hline \multicolumn{4}{|c|}{ Specification no. of intraoperative fluorescent SNs (\% total) [no. of patients] } \\
\hline Visibility through skin & $6(22.2)[2]$ & $11(40.7)[4]$ & $26(35.6)[11]$ \\
\hline \multicolumn{4}{|l|}{ Per basin: } \\
\hline Head & 1 & 1 & 1 \\
\hline Auricular & - & - & 1 \\
\hline Parotid gland & - & - & 2 \\
\hline Neck (level I-V) & 5 & 5 & 17 \\
\hline Axilla & - & 2 & 2 \\
\hline Supraclavicular & - & - & - \\
\hline Scapular & - & 1 & - \\
\hline Groin & - & 2 & 3 \\
\hline Visibility in vivo (prior to excision) & $22(81.4)[6]$ & $27(100)[7]$ & $75(100)[20]$ \\
\hline \multicolumn{4}{|l|}{ Per basin: } \\
\hline Head & 1 & 1 & 5 \\
\hline Auricular & - & - & 1 \\
\hline
\end{tabular}


TABLE 2 continued

\begin{tabular}{|c|c|c|c|}
\hline & \multicolumn{2}{|c|}{ Direct camera comparison } & \multirow[t]{2}{*}{ Evaluation of $\mathrm{m}-\mathrm{PDE}$} \\
\hline & c-PDE & m-PDE & \\
\hline Parotid gland & - & - & 8 \\
\hline Neck (level I-V) & 19 & 21 & 35 \\
\hline Axilla & 1 & 2 & 2 \\
\hline Supraclavicular & - & - & 4 \\
\hline Scapular & 1 & 1 & - \\
\hline Groin & 0 & 2 & 18 \\
\hline Visibility lymphatic duct & - & $2(7.4)[2]$ & $33(45.2)[13]$ \\
\hline \multicolumn{4}{|l|}{ Per basin: } \\
\hline Head & - & - & 2 \\
\hline Auricular & - & - & 1 \\
\hline Parotid gland & - & - & 4 \\
\hline Neck (level I-V) & - & 2 & 15 \\
\hline Axilla & - & - & 2 \\
\hline Supraclavicular & - & - & 4 \\
\hline Scapular & - & - & - \\
\hline Groin & - & - & - \\
\hline \multicolumn{4}{|l|}{ Pathology } \\
\hline No. of tumor-positive SNs (\% total) & $0 / 34$ & & 4/91 (4.4) \\
\hline No. of tumor-positive patients (\% total) & $0 / 7$ & & $4 / 20(20.0)$ \\
\hline
\end{tabular}

$P D E$ Photo Dynamic Eye, SCC squamous cell carcinoma, $M B q$ mega becquerel, $S N$ sentinel node, $S P E C T / C T$ single photon emission computed tomography combined with computed tomography

${ }^{a}$ In two patients blue dye was used. Here 2 SNs were excised of which 1 was blue at the time of excision

${ }^{\mathrm{b}}$ In two patients blue dye was used. Here $2 \mathrm{SNs}$ were excised of which were both blue at the time of excision

\section{Detection Sensitivity Photo Dynamic Eye (PDE) Systems}

Visual inspection of the fluorescence images generated by the m-PDE yielded similar detection sensitivities as reported for the IVIS Spectrum above $\left(1.20 \times 10^{-11} \mathrm{M}\right.$ ICG-HSA) (Fig. 2b) when fluorescence imaging was performed in the dark or under ambient light conditions (surrounding lights and plenum turned on) (Fig. 2b). With all the lights turned on, including the satellite lamps, the fluorescence detection sensitivity for the m-PDE system slightly dropped to $2.40 \times 10^{-11} \mathrm{M}$ ICG-HSA.

With the c-PDE system, a detection sensitivity of $3.08 \times 10^{-9} \mathrm{M}$ ICG-HSA was found under dark conditions (Fig. 2b). This dropped to $4.92 \times 10^{-8} \mathrm{M}$ ICG-HSA when all the lights in the operating room were turned on (Fig. 2b). This two-to-three orders of magnitude difference indicates the m-PDE fluorescence camera system can better cope with the background light present in an intraoperative setting.

\section{Patient Studies}

Conventional PDE Versus Modified PDE Fluorescence Camera System In the comparison study in seven patients (oral cavity cancer $(n=4)$ and melanoma $(n=3))$, a total of 27 SNs were harvested (average 3.9, range 2-7) (Table 2). Initial evaluations performed with the satellite lamps turned on were of limited success and proved to be highly dependent on the positioning of the lamps. For that reason, in this comparison study evaluations were performed with either the satellite lamps dimmed or with these lights turned on, but faced away from the surgical wound bed.

With the m-PDE, under ambient light conditions all SNs evaluated could be easily visualized (100\%). For the cPDE, with all lights in the operating room dimmed an overall detection rate of $81.4 \%$ was found. The m-PDE system visualized $40.7 \%$ of the SNs transcutaneously (11 SNs, 4 patients; ambient light conditions), while the c-PDE system visualized only $22.2 \%$ (6 SNs, 2 patients; dimmed light conditions). In two patients, a lymphatic duct leading to an SN was visualized with the m-PDE (ambient light conditions), whereas no lymphatic ducts could be visualized with the c-PDE (dimmed light conditions). Further detailed results can be found in Table 2 .

Electronic supplementary Fig. SI1 presents the surgical workflow for the c-PDE (Fig. SI1a) and m-PDE (Fig. SI1b) fluorescence camera system. When using the c-PDE (Fig. SI1a), lights in the operating room had to be dimmed in 


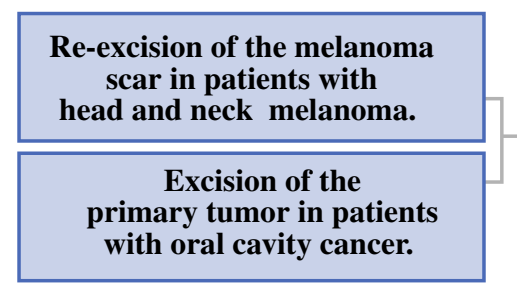

3a. Pre-incision portable gamma
camera imaging to generate an
overview of the area harbouring
the SN(s).
In patients with a melanoma of the trunk or
an extremity, no portable gamma camera
was used.

\section{Evaluation of the preoperatively acquired images. \\ 2. Blue dye injection. No blue dye is used in patients with head and neck melanoma or oral cavity cancer.}

\begin{tabular}{|l|}
\hline $\begin{array}{l}\text { 3b. Pre-incision gamma tracing to } \\
\text { localize the radioactive signal } \\
\text { emitted by the hybrid tracer. }\end{array}$ \\
\hline
\end{tabular}

\section{3c. Pre-incision near-infrared fluorescence imaging to evaluate if the $\mathrm{SN}(\mathrm{s})$ can be visualized through the skin. c-PDE: All lights in the operation room are dimmed prior to fluorscence imaging. \\ m-PDE: Fluorescence imaging is performed under ambient light conditions.}

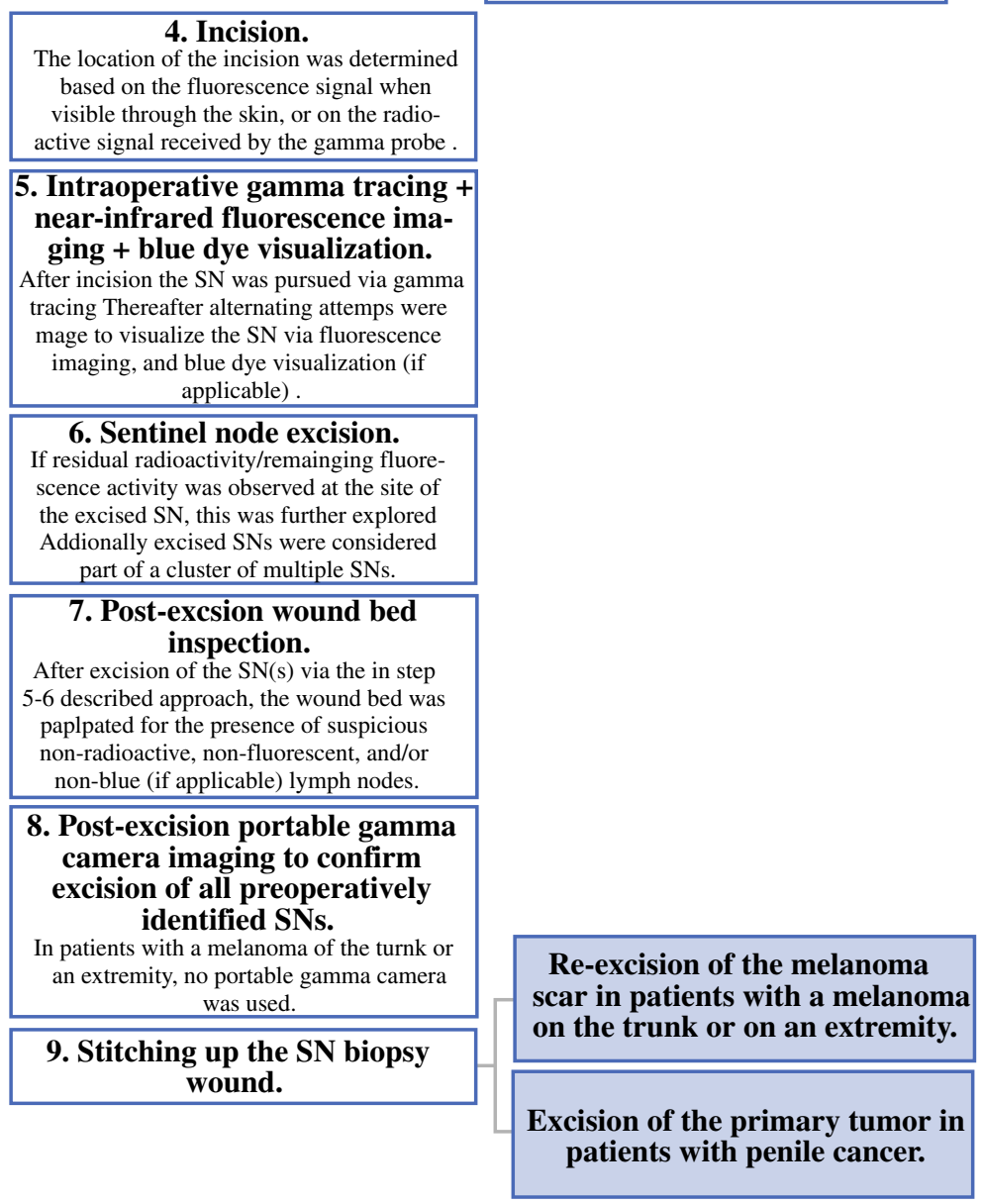

FIG. 1 Workflow for sentinel node localization and excision. Following preoperative image analysis by the surgeon to virtually determine the location of the SNs (1), blue dye can be injected (2). Prior to incision a portable gamma camera (Sentinella; Oncovision, Valencia, Spain), a gamma probe (Neoprobe; Johnson \& Johnson Medical, Hamburg, Germany), and the fluorescence camera (c-PDE or m-PDE; Hamamatsu Photonics K.K., Hamamatsu, Japan) are use to determine the location of the SNs (3). After incision (4) the SN is pursued via gamma tracing, after which alternating attempts were made to visualize the $\mathrm{SN}$ via fluorescence imaging and, when applicable, blue-dye visualization (5). After identification of the SN, the node was excised, after which the wound bed was checked for the presence of residual radioactivity/remaining fluorescence activity at the site of a previously excised SN. Additionally excised nodes were considered part of a cluster of multiple adjacent SNs (6). Following completion of SN biopsy via the combined radio- and fluorescenceguided (and, when applicable, blue dye) approach, the wound-bed was palpated for the presence of suspicious non-radioactive, nonfluorescent and, when applicable, non-blue-dye-stained lymph nodes (8). Thereafter the wound bed was closed (9). SN sentinel node, $P D E$ Photo Dynamic Eye 
A
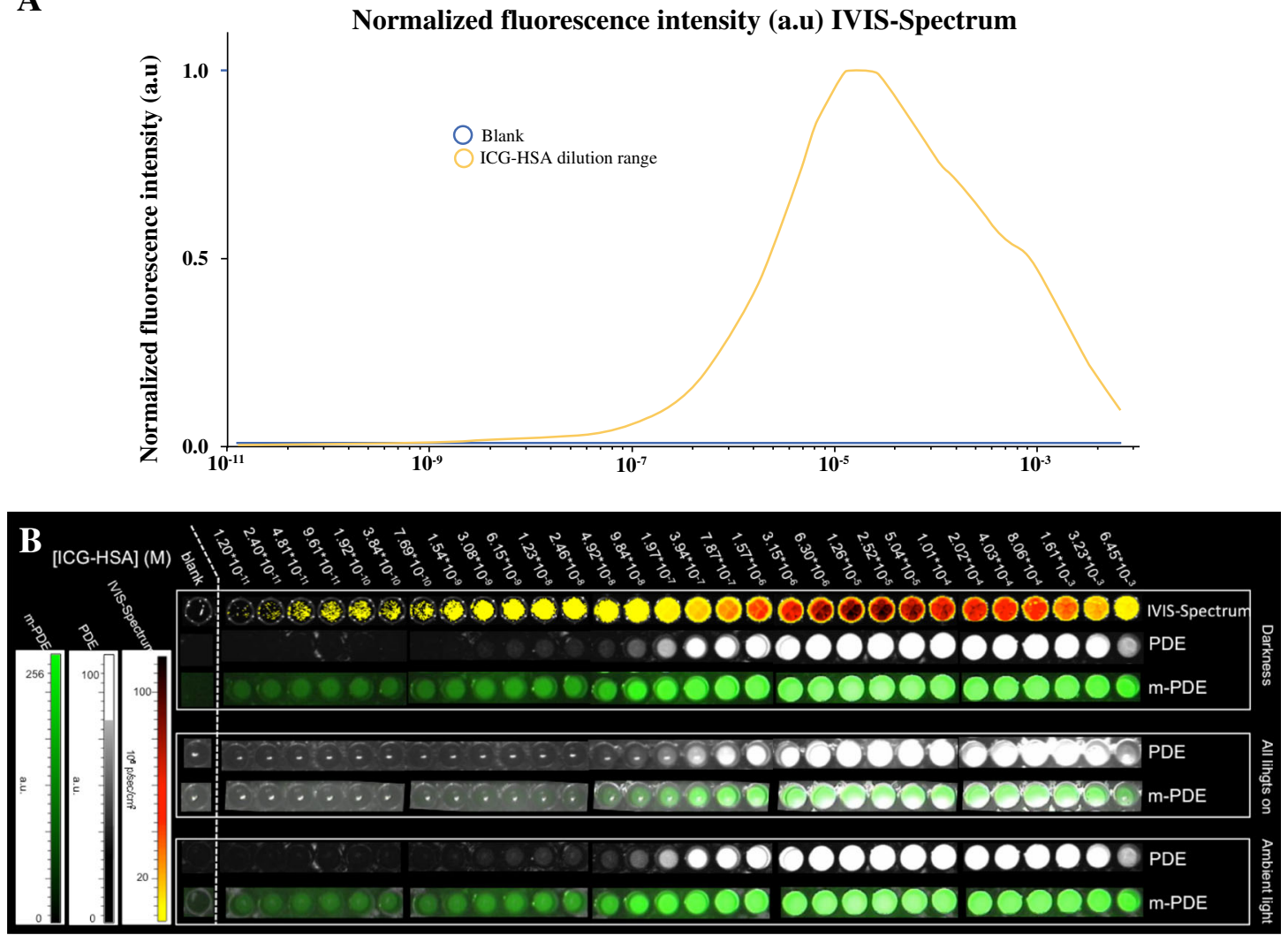

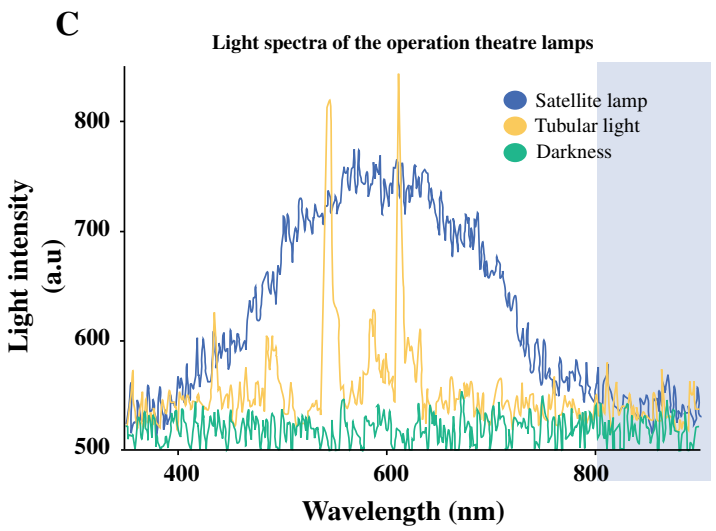

FIG. 2 Determination of the sensitivity of the m-PDE and c-PDE fluorescence camera systems for ICG-HSA. (a) Fluorescence intensity curve of the various steps of the dilution range measured with the IVIS Spectrum. (b) Visual fluorescence images obtained with the IVIS Spectrum, c-PDE, and m-PDE when measured in full darkness, with all lights in the operating room turned on (satellite lamps, plenum, and surrounding lights), and with the satellite lamps directly

order to visualize the SNs. This temporarily stalled the surgical procedure. Forceps were often placed at the location of the SN, after which the lights in the operating room were turned back on to visually confirm the localization of the SN. This was followed by SN excision and fluorescence imaging to confirm removal of the SN. This process was repeated for each individual SN.

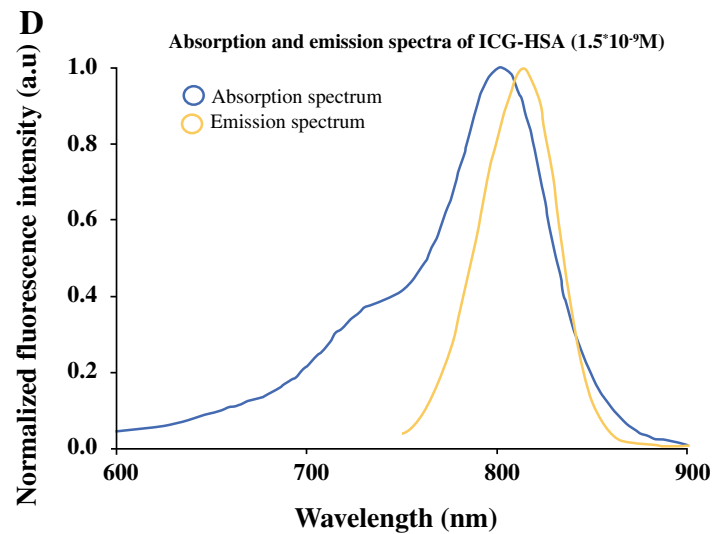

lighting the sterile field turned off, but the plenum and surrounding lights on. (c) Light spectrum of the lamps present in the operating room. The light blue area shows the area in which ICG emits its light. (d) Absorption and emission spectrum of $1.50 \times 10^{-9}$ M ICG-HSA. $I C G$ indocyanine green, HSA human serum albumin, PDE Photo Dynamic Eye

When working with the m-PDE (Fig. SI1b), the presence of ambient light, presentation of the pseudo-colored green fluorescence images on a gray-scaled anatomical background, and the ability to switch the m-PDE to white light mode, combined, allowed the surgeon to directly verify the anatomical location of the SNs and proceed with their excision in a sequential manner. Here, the white light 

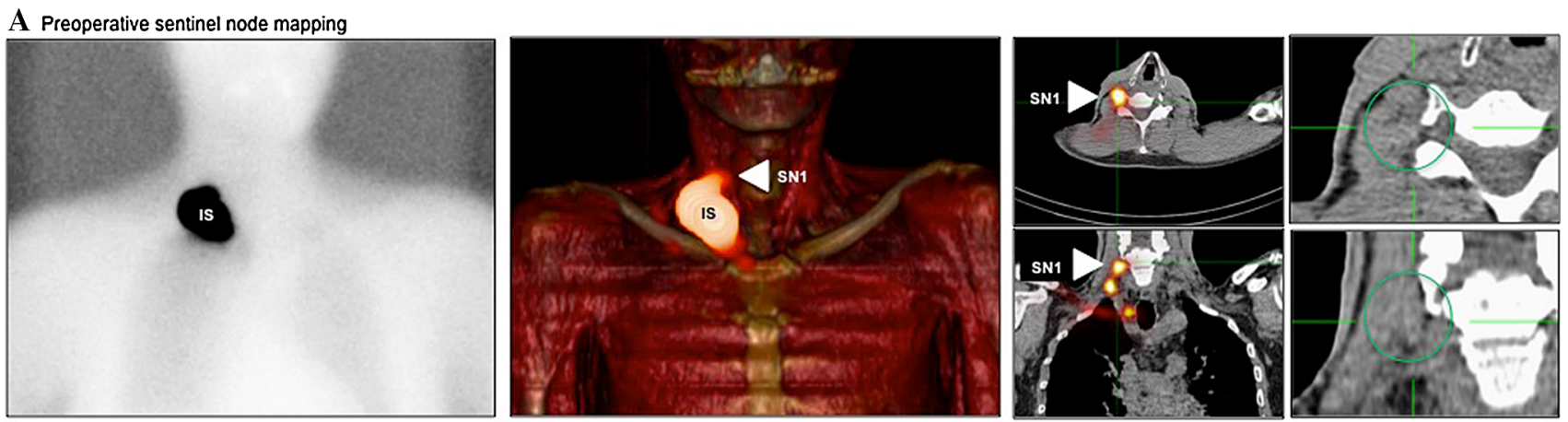

B Intraoperative fluorescence-guided sentinel node identification and excision
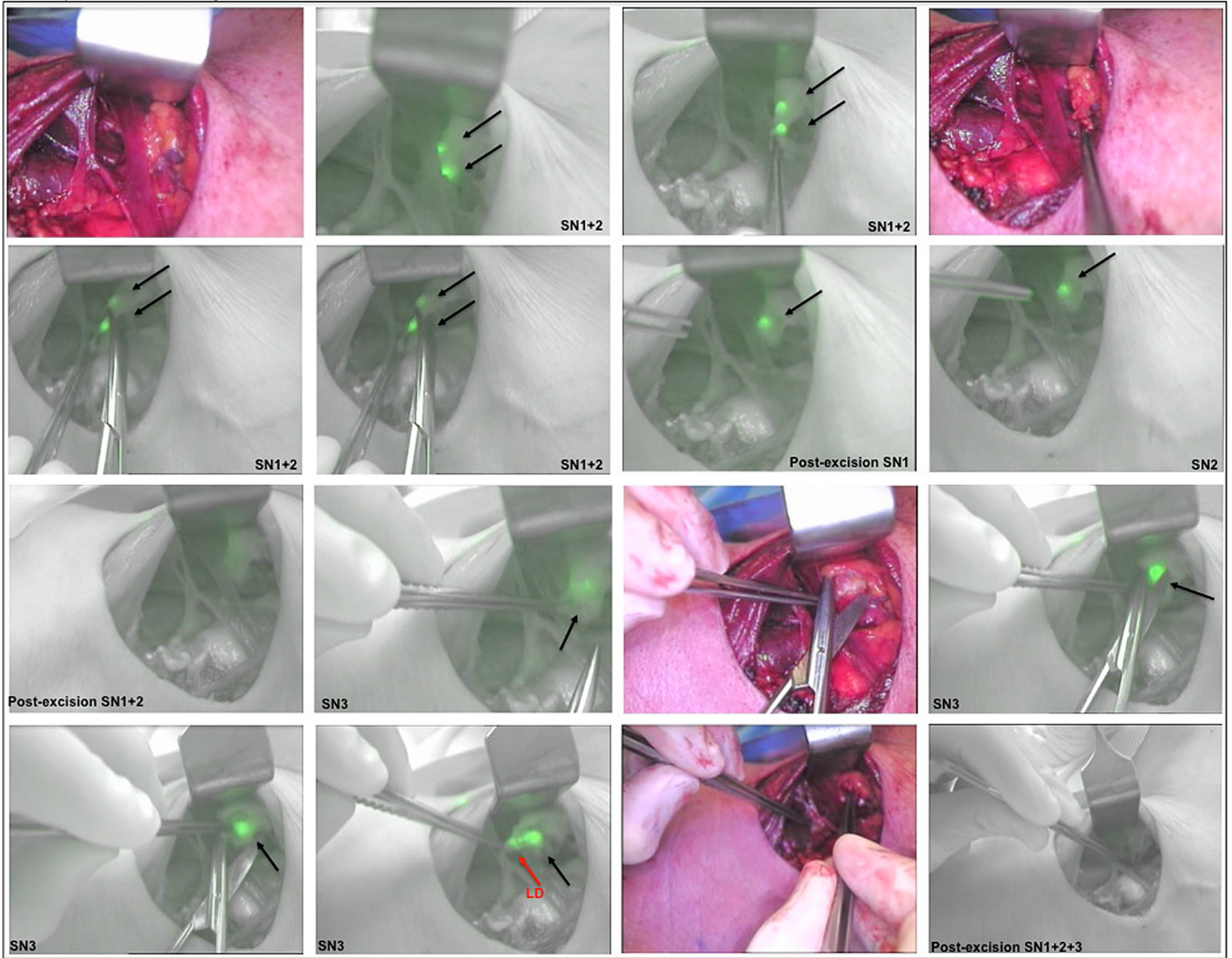

FIG. 3 Fluorescence-guided sentinel node excision in a patient with a melanoma of the neck. (a) Preoperative imaging. Left Static lymphoscintigram acquired $2 \mathrm{~h}$ after hybrid tracer injection showing only the IS. Middle Following fusion of the acquired SPECT and CT images, a 3D volume rendering was generated showing the injection site, as well as an SN in level IV (white arrow) and a supraclavicular SN. Right Axial fused SPECT/CT (left) and CT (right) slice showing the SN in level IV being part of a cluster (indicated because no clear node could be identified on the CT, only a strand of tissue). (b) After re-excision of the melanoma scar, the SN cluster in level IV was pursued via fluorescence imaging using the m-PDE fluorescence camera. The timeline shows fluorescence-guided excision of this cluster of SNs. Switching between the fluorescence and white light image allowed the surgeon to work under continuous fluorescence guidance. A total of three fluorescent (and radioactive) SNs were removed from the area where the hotspot was seen on SPECT/CT imaging. IS injection site, $S N$ sentinel node, SPECT/CT single photon emission computed tomography combined with computed tomography, $3 D$ three-dimensional 
mode allowed us to optimally focus the camera. Please see Fig. 3 for a stepwise illustration on the real-time fluorescence-guided excision of three SNs in a cluster under ambient light conditions. It is interesting to note that even with the increased detection sensitivity of the m-PDE fluorescence camera system, excision of the SNs was not hindered by background signals as a consequence of leakage of tracer from damaged lymphatic ducts (Fig. 3).

\section{Extended Clinical Evaluation of Modified PDE} Fluorescence Camera System The m-PDE fluorescence camera was further evaluated in an additional 20 patients: oral cavity $(n=2)$, penile cancer $(n=5)$, and (head and neck) melanoma $(n=13)$. From these patients, $73 \mathrm{SNs}$ were harvested (average 3.7, range 1-7), of which $35.6 \%$ (26 SNs; 11 patients) could be visualized transcutaneously (Table 2). Lymphatic ducts draining from the primary tumor were identified in 13 patients and 33 SNs (45.2\%) (Table 2). Transcutaneous SN visualization, as well as visualization of the lymphatic ducts, was most pronounced in patients with drainage to SNs in the neck (Table 2). Examples of our findings are shown in Fig. 3 and electronic supplementary Figs. SI2 and SI3.

\section{DISCUSSION}

In the current study, we evaluated the effect that technical improvements have on the performance of the fluorescence camera. For this, the 'new' prototype m-PDE fluorescence camera was evaluated in relation to the 'old' c-PDE. After evaluation in a phantom set-up, its value was defined in patients who were to undergo an SN biopsy procedure for (head and neck) melanoma, oral cavity, or urological malignancies using the hybrid tracer ICG- ${ }^{99 m}$ Tc-nanocollloid. We have previously reported that this hybrid tracer, in combination with the m-PDE's predecessor (the c-PDE), allowed superior optical SN visualization compared to blue dye in, for example, patients with vulvar or penile cancer, ${ }^{8,9}$ or melanoma ${ }^{7}$ (on average, 60.7 versus $96.5 \%$, respectively).

The increased sensitivity of the m-PDE compared with the c-PDE, as concluded from the phantom studies, translated nicely in an improved clinical utility of the m-PDE. In a comparative series of seven patients, the reported two-tothree orders of magnitude increase in detection sensitivity resulted in a $14.8 \%$ increase in $\mathrm{SN}$ visualization. The value of the m-PDE fluorescence camera system was further underlined in 20 additional patients. With the m-PDE, $35.6 \%$ of the SNs could be visualized transcutaneously and, for $45.2 \%$ of the SNs, lymphatic ducts were visualized. Its utility was further enhanced by (1) the fact that the fluorescence image of the m-PDE is corrected real-time for the influence of ambient light, meaning that the lights in the operating theatre did not have to be dimmed when performing fluorescence imaging; (2) the ability of the m-PDE to show the pseudo-colored green fluorescence image on a gray-scale anatomical background image; and (3) its ability to directly switch between the fluorescence light and white light mode. Given the clear clinical potential of this approach for ICG, which is not a particularly bright dye with a relatively short luminescence lifetime, this concept may, in the future, be successfully expanded to other luminescent tracers that have found their way into the clinic. $^{10}$

The technological evolutions realized in the m-PDE help minimize the disturbance of the clinical workflow and help to transform fluorescence imaging from a confirmatory modality to one that provides real-time 'on-screen' guidance during SN excision (as illustrated in Fig. 3 and electronic supplementary Figs. SI1 and SI2). This optimized 'on-screen' guidance set-up is comparable to the type of guidance obtained during (fluorescence-guided) laparoscopic surgery. ${ }^{4,11}$ However, during open surgery procedures, the small overlap of the ICG light spectrum and the light emitted by the satellite lamp (Fig. 2), in combination with the high intensity of this light source (Fig. 2), still meant that the satellite lamps had to be faced away from the surgical wound bed (or turned off) for optimal guidance. With the upcoming modernized operating rooms, in which halogen satellite lamps are exchanged for LED lamps, this effect will likely become less prominent.

In the current study, we evaluated the m-PDE in combination with ICG- ${ }^{-99 \mathrm{~m}} \mathrm{Tc}$-nanocollloid, a hybrid tracer that was specifically designed as an SN tracer. ${ }^{12,13}$ The specificity of this tracer was further confirmed by the minimal leakage from the lymphatic ducts that we observed with the m-PDE (Fig. 3). When compared with other studies using 'free' ICG where such leakage is more common, ${ }^{11}$ this outcome underlines the advantage of using an $\mathrm{SN}$-specific tracer for SN biopsy procedures. From a technical perspective, the advantages the m-PDE has can, in the future, also provide value in applications for which 'free' ICG is used, e.g. during angiography applications such as free-flap reconstruction ${ }^{14}$ or partial nephrectomy, ${ }^{15}$ for lymphedema imaging, ${ }^{16}$ lymphatic mapping ${ }^{11}$ or the identification of postoperative lymphatic leaks, ${ }^{17}$ or the for the identification of metastases in the liver. ${ }^{18}$

\section{CONCLUSION}

The m-PDE fluorescence camera system enhances the fluorescence imaging properties and simplifies the workflow compared with its predecessor. We thus think it 
provides a critical next step in the routine use of fluorescence-guided surgery.

ACKNOWLEDGMENTS The authors gratefully acknowledge the entire surgical staff of The Netherlands Cancer Institute-Antoni van Leeuwenhoek Hospital for their assistance.

DISCLOSURES This work was partially supported by a Dutch Cancer Society translational research award (Grant No. PGF 20094344), an NWO-STW-VIDI Grant (Grant No. STW BGT11272), and a European Research Council under the European Union's Seventh Framework Program (FP7/2007-2013) Grant (Grant No. 2012306890). The study was supported by Hamamatsu Photonics K.K., in providing the $\mathrm{m}-\mathrm{PDE}$ fluorescence camera that was developed for the study.

OPEN ACCESS This article is distributed under the terms of the Creative Commons Attribution 4.0 International License (http:// creativecommons.org/licenses/by/4.0/), which permits unrestricted use, distribution, and reproduction in any medium, provided you give appropriate credit to the original author(s) and the source, provide a link to the Creative Commons license, and indicate if changes were made.

\section{REFERENCES}

1. Crane LM, Themelis G, Arts HJ, Buddingh KT, Brouwers AH, Ntziachristos $\mathrm{V}$, et al. Intraoperative near-infrared fluorescence imaging for sentinel lymph node detection in vulvar cancer: first clinical results. Gynecol Oncol. 2011;120(2):291-5.

2. Mieog JS, Troyan SL, Hutteman M, Donohoe KJ, van der Vorst JR, Stockdale A, et al. Toward optimization of imaging system and lymphatic tracer for near-infrared fluorescent sentinel lymph node mapping in breast cancer. Ann Surg Oncol. 2011; 18(9):2483-91.

3. Kusano M, Kokudo N, Toi M, Kaibori M (eds). ICG fluorescence imaging and navigation surgery. New York: Springer; 2016.

4. KleinJan GH, van den Berg NS, Brouwer OR, de Jong J, Acar C, Wit EM, et al. Optimisation of fluorescence guidance during robot-assisted laparoscopic sentinel node biopsy for prostate cancer. Eur Urol. 2014;66(6):991-8.

5. Brouwer OR, Klop WM, Buckle T, Vermeeren L, van den Brekel MW, Balm AJ, et al. Feasibility of sentinel node biopsy in head and neck melanoma using a hybrid radioactive and fluorescent tracer. Ann Surg Oncol. 2012;19(6):1988-94.

6. van den Berg NS, Brouwer OR, Klop WM, Karakullukcu B, Zuur $\mathrm{CL}$, Tan IB, et al. Concomitant radio- and fluorescence-guided sentinel lymph node biopsy in squamous cell carcinoma of the oral cavity using ICG-(99m)Tc-nanocolloid. Eur J Nucl Med Mol Imaging. 2012;39(7):1128-36.
7. van den Berg NS, Brouwer OR, Schaafsma BE, Matheron HM, Klop WM, Balm AJ, et al. Multimodal surgical guidance during sentinel node biopsy for melanoma: combined gamma tracing and fluorescence imaging of the sentinel node through use of the hybrid tracer indocyanine green- $(99 \mathrm{~m}) \mathrm{Tc}-$ nanocolloid. Radiology. 2015;275(2):521-9.

8. Brouwer OR, van den Berg NS, Matheron HM, van der Poel HG, van Rhijn BW, Bex A, et al. A hybrid radioactive and fluorescent tracer for sentinel node biopsy in penile carcinoma as a potential replacement for blue dye. Eur Urol. 2014;65(3):600-9.

9. Matheron HM, van den Berg NS, Brouwer OR, Kleinjan GH, van Driel WJ, Trum JW, et al. Multimodal surgical guidance towards the sentinel node in vulvar cancer. Gynecol Oncol. 2013;131(3): $720-5$.

10. van Leeuwen FW, Hardwick JC, van Erkel AR. Luminescencebased imaging approaches in the field of interventional molecular imaging. Radiology. 2015;276(1):12-29.

11. Manny TB, Patel M, Hemal AK. Fluorescence-enhanced robotic radical prostatectomy using real-time lymphangiography and tissue marking with percutaneous injection of unconjugated indocyanine green: the initial clinical experience in 50 patients. Eur Urol. 2014;65(6):1162-8.

12. Van Den Berg NS, Buckle T, Kleinjan GI, Klop WM, Horenblas $\mathrm{S}$, Van Der Poel HG, et al. Hybrid tracers for sentinel node biopsy. Q J Nucl Med Mol Imaging. 2014;58(2):193-206.

13. Brouwer OR, Buckle T, Vermeeren L, Klop WM, Balm AJ, van der Poel HG, et al. Comparing the hybrid fluorescent-radioactive tracer indocyanine green-99mTc-nanocolloid with $99 \mathrm{mTc}-$ nanocolloid for sentinel node identification: a validation study using lymphoscintigraphy and SPECT/CT. J Nucl Med. 2012;53(7):1034-40.

14. Holm C, Dornseifer U, Sturtz G, Basso G, Schuster T, Ninkovic M. The intrinsic transit time of free microvascular flaps: clinical and prognostic implications. Microsurgery. 2010;30(2):91-6.

15. Bjurlin MA, Gan M, McClintock TR, Volpe A, Borofsky MS, Mottrie A, et al. Near-infrared fluorescence imaging: emerging applications in robotic upper urinary tract surgery. Eur Urol. 2014;65(4):793-801.

16. Unno N, Nishiyama M, Suzuki M, Tanaka H, Yamamoto N, Sagara D, et al. A novel method of measuring human lymphatic pumping using indocyanine green fluorescence lymphography. $J$ Vasc Surg. 2010;52(4):946-52.

17. Tan IC, Balaguru D, Rasmussen JC, Guilliod R, Bricker JT, Douglas WI, et al. Investigational lymphatic imaging at the bedside in a pediatric postoperative chylothorax patient. Pediatr Cardiol. 2014;35(7):1295-300.

18. Ishizawa T, Fukushima N, Shibahara J, Masuda K, Tamura S, Aoki T, et al. Real-time identification of liver cancers by using indocyanine green fluorescent imaging. Cancer. 2009;115(11): 2491-504. 